Vol. 4, No. 2, 2018

Ihor Kuzio ${ }^{1}$, Viktor Zakharov ${ }^{2}$, Vitaliy Korendiy ${ }^{3}$

1. Department of Mechanics and Automation Engineering, Lviv Polytechnic National University, Ukraine, Lviv,

S. Bandery street 12, E-mail: ihor.v.kuzo@lpnu.ua

2. ISKRA PJSC, Ukraine, Lviv, Vuletska Street 14, E-mail: vktzakharov@gmail.com

3. Department of Mechanics and Automation Engineering, Lviv Polytechnic National University, Ukraine, Lviv,

S. Bandery street 12, E-mail: vitalii.m.korendii@lpnu.ua

\title{
SUBSTANTIATION OF INERTIAL, STIFFNESS AND EXCITATION PARAMETERS OF VIBRATORY LAPPING MACHINE WITH LINEAR OSCILLATIONS OF LAPS
}

Received: August 20, 2018 / Revised: September 29, 2018 / Accepted: December 26, 2018

(C) Kuzio I., Zakharov V., Korendiy V., 2018

\begin{abstract}
Problem statement. Designing and manufacturing of efficient resonant vibratory lapping machines with linear oscillations of laps demand an accurate and detailed calculation of parameters of their elastic systems and electromagnetic drives. Purpose. The main objective of this research consists in derivation of analytical dependencies for calculating the stiffness and excitation parameters of mechanical oscillatory system of vibratory finishing machine in order to ensure its resonance operation mode. Methodology. The technique of the research is based on fundamental concepts of engineering mechanics, strength of materials and theory of mechanical vibrations. Findings (results). The design diagram of mechanical oscillatory system of vibratory finishing machine with linear oscillations of laps is considered and corresponding equations of motion are presented. Analytical dependencies for calculating stiffness and excitation parameters of the system are deduced. The example of parameters calculation is given and time dependencies of the system's motion are constructed. Originality (novelty). The mathematical model of linear oscillations of the three-mass mechanical system of vibratory finishing machine was developed. The possibilities of performing the laps dressing using "lap over lap" method were substantiated. Practical value. The results of the performed investigations can be used during designing new designs and improving existing structures of vibratory finishing machines for lapping flat surfaces of cylindric and prismatic parts. Scopes of further investigations. In further investigations, it is necessary to analyse the influence of the viscous damping on the system's motion. In order to substantiate (justify) the obtained theoretical results, the experimental investigations should be carried out.
\end{abstract}

Keywords: lapping, finishing treatment, vibratory machine, electromagnetic drive, linear oscillations, equations of motion, stiffness parameters, excitation parameters.

\section{Introduction}

In mechanical engineering, the technological operations of finishing treatment occupy about $20-30 \%$ of the total duration of machine parts treatment. The productiveness, efficiency and accuracy of finishing operation depend on the technological equipment being used. It is known, that the surface quality of the parts treated by the lapping techniques is usually higher than that of the parts treated by grinding, superfinishing or honing. Thus, designing, investigating, manufacturing and implementation of highperformance lapping machines are the necessary factors of development of modern technologies of finishing treatment at major mechanical engineering enterprises.

\section{Problem Statement}

Resonant vibratory lapping machine with circular oscillations of laps is one of the most efficient finishing equipment. Such machines are used for high-accuracy single- and double-sided finishing 


\section{Substantiation of Inertial, Stiffness and Excitation Parameters of Vibratory Lapping Machine ...}

treatment of flat surfaces of machine parts. This equipment provide the uniform removal of a material layer from parts' surfaces and can treat metal, plastic, and ceramic workpieces.

Designing and manufacturing of efficient resonant vibratory lapping machines with linear oscillations of laps is the first step of creating more complicated equipment (for example, vibratory finishing machines with circular oscillations of the working bodies). The processes of development of such machines demand an accurate and detailed calculation of parameters of their elastic systems and electromagnetic drives. The operational efficiency of vibratory lapping machine with linear oscillations of laps essentially depends on the accuracy of calculation of its stiffness and excitation parameters, which ensure the energy-efficient resonance operation mode of the machine.

\section{Review of Modern Information Sources on the Subject of the Paper}

The paper [1] presents the general overview of the process of vibratory finishing treatment and analyses the modern state of scientific research on this subject. There is concluded that the problems of calculating optimal frequencies and amplitudes of vibrations of the working elements of vibratory finishing machines, of analysing the influence of the speed of displacement of the parts with respect to the abrasive media on the accuracy of parts treatment and on the quality of surfaces being treated are not enough investigated. In the publication [2], the authors proposed a new approach to designing and modelling the dynamic characteristics of ultraprecision micro-milling machines. The authors analysed the dynamics of the machine's structure and the kinematics of moving components; they also overviewed the control system and the machining process. The results of these investigations can be used to predict the overall machine performance. The new method of vibratory surface grinding of machine parts made of mild and hardened steel is proposed in [3]. The authors developed the design of corresponding grinding equipment which ensure such grinding process parameters (frequency and amplitude of oscillations, trajectory of the treated workpiece motion) providing the increase of the efficiency of the process, reducing the energy consumption and improving the quality of the surfaces being treated.

In the work [4], there is considered the process of double-side lapping of cylindrical surfaces with a help of planetary mechanism used in the machine's drive. The analysis of influence of the rolling speed of rollers on the quality of the treated surface is carried out. On the basis of this analysis, the optimal geometry and motion kinematics of parts are described. The process of abrasive lapping of plates made of copper and stainless steel is investigated in [5]. The kinematics of the plates' motion during their treatment is considered. The uniformity of the surfaces lapping was assessed depending on the speed of the workpieces' motion and on the force of their pressing to the working surfaces of laps. The paper [6] analyses the basic principles of vibratory finishing treatment of machine parts. The authors developed the mathematical models, which allow predicting the parts' surface roughness and stock removal intensity. The experimental results substantiated the validity of the theoretical modelling. This allowed developing the algorithm of designing an optimum process of vibratory finishing treatment.

In the paper [7], there was developed a mathematical model of the bowl-type vibratory finishing machine. This model was used for analysing the dynamic behaviour of the machine's mechanical oscillatory system considering both free and forced vibrations. The influence of the oscillatory system's parameters on the machine performance was studied. The comparative analysis of theoretical and experimental results was carried out. In the study [8], the medium kinematic model was developed and its movement simulation was performed. Based on the kinematic model, the material removal intensity model was further established in order to better understand the vibratory finishing process. The optimal fixing location and orientation of the workpiece were substantiated by analysing the kinematic model. The theoretical results were justified be the experimental studies. In [9], there is presented the analysis of onedimensional controlled vibrations of vibratory finishing machine. The obtained results covers the following characteristics: material removal rates, surface roughness and contact forces between the surfaces being treated and the working media. The media motion was modelled and experimentally tested. This work is the first step before carrying out further three-dimensional simulation of vibratory finishing process. 


\section{Ihor Kuzio, Viktor Zakharov, Vitaliy Korendiy}

Analysing the information sources on the subject of vibratory finishing treatment of flat surfaces of cylindrical and prismatic parts, we can state that the problems of dynamic analysis, substantiation of parameters and operation modes, as well as simulation modelling of vibratory lapping machines operation are not completely solved. In the papers [10]-[12], the investigations of the authors on the mentioned subject were initiated. In particular, three- and four-mass two-dimensional and three-dimensional diagrams of vibratory finishing machines were considered, the differentials equations of the working bodies' motion were derived, inertial and stiffness parameters of corresponding mechanical oscillatory systems were substantiated, and simulation modelling of the oscillatory motion of laps during their dressing was carried out. In this paper, the investigations initiated in [10]-[12] will be completed in the following directions: developing a two-dimensional design (calculation) diagram of three-mass mechanical oscillatory system of vibratory finishing machine; deriving the corresponding differential equations of the oscillating masses' motion; substantiating the inertial and stiffness parameters and the parameters of excitation of the system; dynamic analysis of periodical one-dimensional motion (linear oscillations) of the laps during their dressing; simulation modelling of the laps' motion etc.

\section{Objectives and Problems of Research}

The main purpose of the presented paper consists in derivation of analytical dependencies for calculating the stiffness and excitation parameters of mechanical oscillatory system of vibratory finishing machine in order to ensure its resonance operation mode.

With the object of satisfying the stated purpose, the following tasks will be solved: the design diagram of mechanical oscillatory system of vibratory finishing machine with linear oscillations of laps will be considered; the corresponding equations of motion will be presented; the analytical dependencies for calculating stiffness and excitation parameters of the system will be deduced; the example of parameters calculation will be given; time dependencies of the system's motion will be constructed.

\section{Main Material Presentation}

In order to perform further modelling of the laps' dressing of vibratory finishing machine using the "lap over lap" method, let us consider the simplified design (calculation) diagram of its mechanical oscillatory system (Fig. 1). The body of the mass $m_{1}$ is the upper lap, which can slide over the working surface of the lower lap (the body of the mass $m_{2}$ ) and is kinematically connected to the lower lap with a help of the elastic system characterized by the stiffness $c_{1}$ and damping coefficient $\mu_{1}$. The lower lap is mounted (installed) on the unmovable supporting surface (foundation, frame, body etc.) using the vibration isolators with the characteristics of stiffness and damping $c_{i 3}$ and $\mu_{i 3}$, respectively. The excitation of the system's oscillations is performed due to application (exerting) of the periodically changing force $F(t)$ between the reactive mass $m_{3}$ and the lower lap. Herewith, the reactive mass is connected to the lower lap with a help of the elastic elements with stiffness $c_{2}$ and damping coefficient $\mu_{2}$. The application of the periodically changing force $F(t)$ between the reactive mass $m_{3}$ and the lower lap $m_{2}$ provides the excitation of periodical linear motion (linear oscillations) of the laps with respect to each other. In the conditions when the phases of the laps' oscillations are not equal, the process of their mutual (reciprocal) dressing (or, so called, process of "lap over lap" dressing) will be performed. This process will be the most intensive in the conditions of antiphase motion of the laps.

Therefore, the one of the major problems (tasks) of this paper consists in substantiation of the stiffness and excitation parameters of the mechanical oscillatory system of vibratory lapping machine in order to provide the antiphase oscillations of the laps.

To describe the motion of the oscillating masses, let us take the inertial coordinate system with the centre at point $O$ (Fig. 1). Herewith, the axis $O y$ is vertical (directed upwards), and $O x$ is a horizontal axis, in parallel to which the oscillations of the system's bodies are performed. The motion of the 

oscillating masses $m_{1}, m_{2}$ and $m_{3}$ will be described by the corresponding generalized coordinates $x_{1}, x_{2}$ and $x_{3}$, which represent the displacements of the mass centres of the upper lap, lower lap and reactive (exciting) mass with respect to their equilibrium positions.

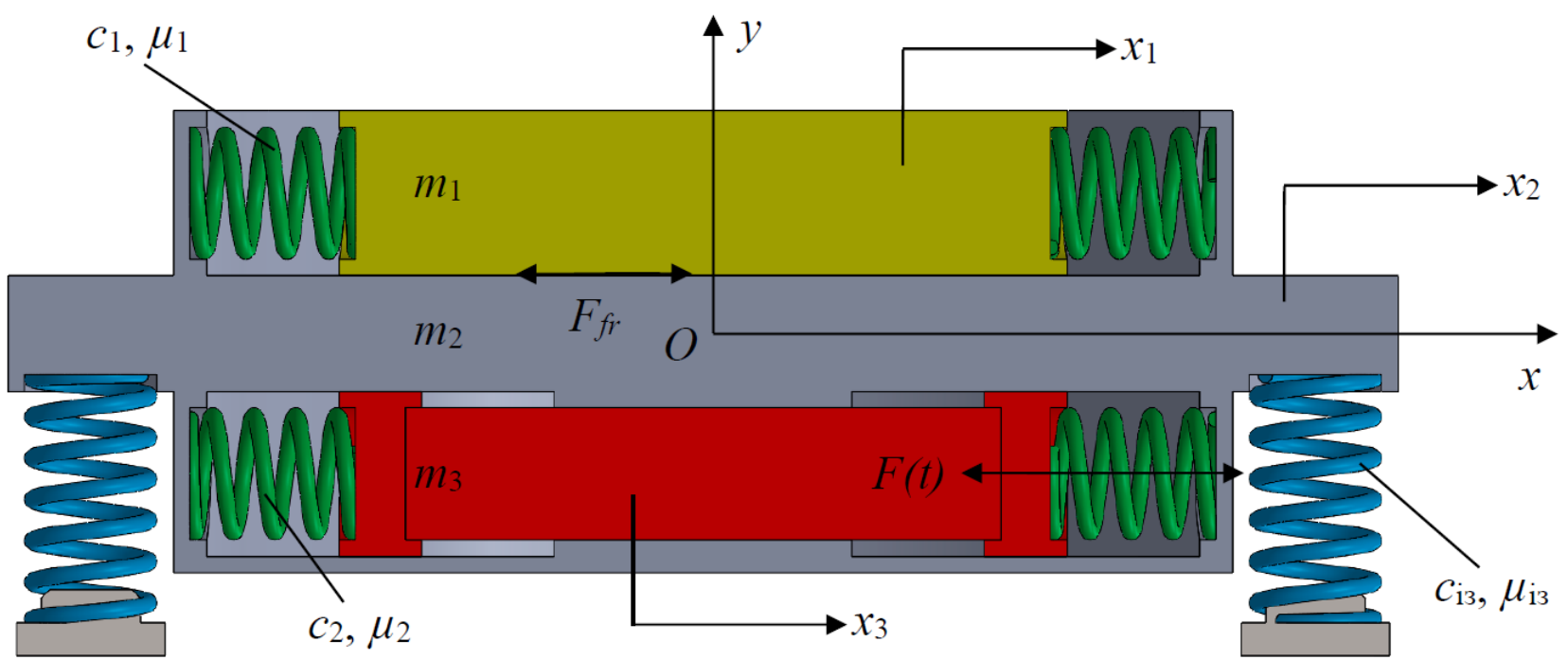

Fig. 1. Design diagram of mechanical oscillating system of vibratory finishing machine

In the plane of contact of the laps' working surfaces, there occurs the friction force, which will be modelled in accordance with the Amontons'-Coulomb's laws. Also, let us assume that the deformations of the elastic elements are performed according to the Hooke's law, and the energy dissipation is proportional to the square of the speed of their deformation.

The mathematical model of the vibratory finishing machine, the calculation diagram of which is presented in Fig. 1, is developed on the basis of the three-mass mechanical oscillatory system, in which the linear oscillations of the masses are performed in horizontal plane. The active $1\left(m_{1}\right)$, intermediate $2\left(m_{2}\right)$ and reactive $3\left(m_{3}\right)$ masses perform linear oscillations along the horizontal axis $x$ described by the generalized coordinates $x_{1}, x_{2}$ and $x_{3}$ due to the influence of the disturbing force $F(t)$. The system of differential equations of the three-mass system's motion has the following form:

$$
\left\{\begin{array}{c}
m_{1} \cdot \ddot{x}_{1}+c_{1} \cdot\left(x_{1}-x_{2}\right)+\left(\mu_{1}+\mu_{t}\right) \cdot\left(\dot{x}_{1}-\dot{x}_{2}\right)=-F_{f r} \cdot \operatorname{sign}\left(\dot{x}_{1}-\dot{x}_{2}\right) ; \\
m_{2} \cdot \ddot{x}_{2}+c_{1} \cdot\left(x_{2}-x_{1}\right)+c_{2} \cdot\left(x_{2}-x_{3}\right)+c_{13} \cdot x_{2}+\left(\mu_{1}+\mu_{t}\right) \cdot\left(\dot{x}_{2}-\dot{x}_{1}\right)+\mu_{2} \cdot\left(\dot{x}_{2}-\dot{x}_{3}\right)+ \\
+\mu_{13} \cdot \dot{x}_{2}=F \cdot \sin (\omega t+\varepsilon)+F_{f r} \cdot \operatorname{sign}\left(\dot{x}_{1}-\dot{x}_{2}\right) ; \\
m_{3} \cdot \ddot{x}_{3}+c_{2} \cdot\left(x_{3}-x_{2}\right)+\mu_{2} \cdot\left(\dot{x}_{3}-\dot{x}_{2}\right)=-F \cdot \sin (\omega t+\varepsilon),
\end{array}\right.
$$

where $\mu_{t}$ is the damping coefficient (coefficient of viscous friction) caused by technological media located between the laps' surfaces; $F$ is the amplitude value of the excitation force; $\omega$ is the forced circular frequency; $\varepsilon$ is the initial phase of the excitation force; $F_{f r}=f \cdot m_{1} \cdot g \cdot \operatorname{sign}\left(\dot{x}_{1}(t)-\dot{x}_{2}(t)\right)$ is the friction force between the laps' contacting surfaces; $f$ is the coefficient of sliding friction depending on the materials and on the state of the laps' working surfaces; $g$ is free-fall acceleration; $\operatorname{sign}\left(\dot{x}_{1}(t)-\dot{x}_{2}(t)\right)$ is the function defining (specifying) the direction of the mutual (reciprocal) motion of the upper and lower laps. It can be determined by the following formula:

$$
\operatorname{sign}\left(\dot{x}_{1}(t)-\dot{x}_{2}(t)\right)=\left\{\begin{array}{l}
1, \text { if }\left(\dot{x}_{1}(t)-\dot{x}_{2}(t)\right)>0 ; \\
-1, \text { if }\left(\dot{x}_{1}(t)-\dot{x}_{2}(t)\right)<0 \\
0, \text { if }\left(\dot{x}_{1}(t)-\dot{x}_{2}(t)\right)=0 .
\end{array}\right.
$$




\section{Ihor Kuzio, Viktor Zakharov, Vitaliy Korendiy}

The obtained system (1) is a system of linear inhomogeneous differential equations of the second order with constant coefficients. In order to determine eigenfrequencies of the mechanical system it is necessary to define the general solutions of the corresponding system of homogeneous equations:

$$
\left\{\begin{array}{c}
m_{1} \cdot \ddot{x}_{1}+c_{1} \cdot\left(x_{1}-x_{2}\right)+\left(\mu_{1}+\mu_{t}\right) \cdot\left(\dot{x}_{1}-\dot{x}_{2}\right)=0 ; \\
m_{2} \cdot \ddot{x}_{2}+c_{1} \cdot\left(x_{2}-x_{1}\right)+c_{2} \cdot\left(x_{2}-x_{3}\right)+c_{13} \cdot x_{2}+\left(\mu_{1}+\mu_{t}\right) \cdot\left(\dot{x}_{2}-\dot{x}_{1}\right)+\mu_{2} \cdot\left(\dot{x}_{2}-\dot{x}_{3}\right)+\mu_{\mathrm{i} 3} \cdot \dot{x}_{2}=0 ; \\
m_{3} \cdot \ddot{x}_{3}+c_{2} \cdot\left(x_{3}-x_{2}\right)+\mu_{2} \cdot\left(\dot{x}_{3}-\dot{x}_{2}\right)=0 .
\end{array}\right.
$$

Using the general methods of solving the obtained system of differential equations (1), let us define the analytical expressions of the masses motion by three degrees of freedom in the following form:

$$
x_{1}=X_{1} \cdot e^{i \cdot \omega \cdot t} ; \quad x_{2}=X_{2} \cdot e^{i \cdot \omega \cdot t} ; \quad x_{3}=X_{3} \cdot e^{i \cdot \omega \cdot t},
$$

where $i=\sqrt{-1} ; \omega$ is the circular frequency of the system's vibrations; $X_{1}, X_{2}, X_{3}$ are the amplitude values of linear forced oscillations by the generalized coordinates $x_{1}, x_{2}, x_{3}$, respectively.

Taking the corresponding derivatives of equations (3) with respect to time and substituting the obtained expressions into the system of equations (2), after cancelling the term $e^{i \cdot \omega \cdot t}$, we obtain:

$$
\left\{\begin{array}{c}
-m_{1} \cdot \omega^{2} \cdot X_{1}+c_{1} \cdot\left(X_{1}-X_{2}\right)+\left(\mu_{1}+\mu_{t}\right) \cdot\left(\omega \cdot i \cdot X_{1}-\omega \cdot i \cdot X_{2}\right)=0 ; \\
m_{2} \cdot \omega^{2} \cdot X_{2}+c_{1} \cdot\left(X_{2}-X_{1}\right)+c_{2} \cdot\left(X_{2}-X_{3}\right)+c_{\mathrm{i} 3} \cdot X_{2}+\left(\mu_{1}+\mu_{t}\right) \cdot\left(\omega \cdot i \cdot X_{2}-\omega \cdot i \cdot X_{1}\right)+ \\
+\mu_{2} \cdot\left(\omega \cdot i \cdot X_{2}-\omega \cdot i \cdot X_{3}\right)+\mu_{\mathrm{i} 3} \cdot \omega \cdot i \cdot X_{2}=0 ; \\
m_{3} \cdot \omega^{2} \cdot X_{3}+c_{2} \cdot\left(X_{3}-X_{2}\right)+\mu_{2} \cdot\left(\omega \cdot i \cdot X_{3}-\omega \cdot i \cdot X_{2}\right)=0 .
\end{array}\right.
$$

Let us reduce the obtained system (4) of linear algebraic equations with constant coefficients to the normal form with respect to the constants $X_{1}, X_{2}, X_{3}$ :

$$
\begin{gathered}
\left(-m_{1} \cdot \omega^{2}+\left(\mu_{1}+\mu_{t}\right) \cdot i \cdot \omega+c_{1}\right) \cdot X_{1}-\left(c_{1}+\left(\mu_{1}+\mu_{t}\right) \cdot i \cdot \omega\right) \cdot X_{2}=0 \\
-\left(\left(\mu_{1}+\mu_{t}\right) \cdot i \cdot \omega+c_{1}\right) \cdot X_{1}+\left(-m_{2} \cdot \omega^{2}+\left(\mu_{1}+\mu_{2}+\mu_{i 3}+\mu_{t}\right) \cdot i \cdot \omega+c_{1}+c_{2}+c_{i 3}\right) \cdot X_{2}- \\
-\left(\mu_{2} \cdot i \cdot \omega+c_{2}\right) \cdot X_{3}=0 ; \\
-\left(\mu_{2} \cdot i \cdot \omega+c_{2}\right) \cdot X_{2}+\left(-m_{3} \cdot \omega^{2}+\mu_{2} \cdot i \cdot \omega+c_{2}\right) \cdot X_{3}=0 .
\end{gathered}
$$

Taking into account the expression (5), let us write the matrix form of the system of equations (1) neglecting dry friction between the working surfaces of laps:

$$
\left[\begin{array}{cc}
\left(\begin{array}{c}
-m_{1} \cdot \omega^{2}+ \\
+\left(\mu_{1}+\mu_{t}\right) \cdot i \cdot \omega+c_{1}
\end{array}\right) & -\left(c_{1}+\left(\mu_{1}+\mu_{t}\right) \cdot i \cdot \omega\right) \\
-\left(c_{1}+\left(\mu_{1}+\mu_{t}\right) \cdot i \cdot \omega\right) & 0 \\
+\left(\mu_{1}+\mu_{2}+\mu_{i 3}+\mu_{t}\right) \cdot i \cdot \omega+ \\
+c_{1}+c_{2}+c_{i 3} \\
-\left(\mu_{2} \cdot i \cdot \omega+c_{2}\right) & -\left(\mu_{2} \cdot i \cdot \omega+c_{2}\right) \\
0 & \left(\begin{array}{c}
-m_{3} \cdot \omega^{2}+ \\
+\mu_{2} \cdot i \cdot \omega+c_{2}
\end{array}\right)
\end{array}\right] \times\left[\begin{array}{c}
X_{1} \\
X_{2} \\
X_{3}
\end{array}\right]=\left[\begin{array}{c}
0 \\
F \\
-F
\end{array}\right] .
$$

The constants $X_{1}, X_{2}, X_{3}$ are not equal to zero only in the case when the determinant of the system (6) equals zero: 
Substantiation of Inertial, Stiffness and Excitation Parameters of Vibratory Lapping Machine ...

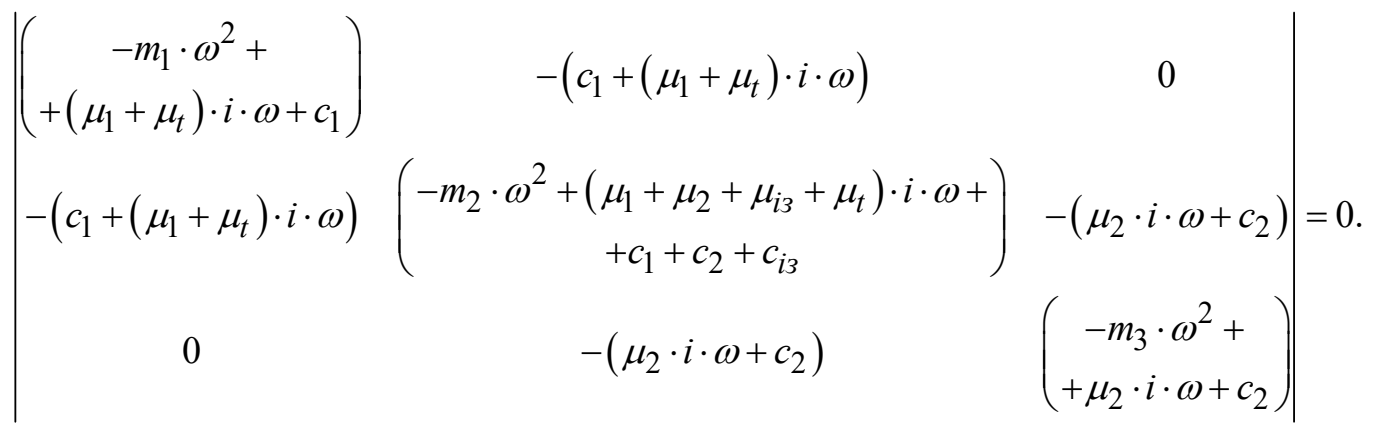

The equation (7) is a characteristic equation for the system (2). It may be reduced to one power equation with respect to $\omega$. Since the length of the obtained equations, as well as their complexity, is very large, in major publications (for example, $[6,7,11-14]$ ) the authors recommend to neglect the system's damping parameters $\left(\mu_{1}=\mu_{2}=\mu_{i 3}=\mu_{t}=0\right)$ and the stiffness of vibration isolators. The last assumption can be explained by the fact that the magnitude of stiffness coefficients of vibration isolators is much smaller than that of the basic elastic elements of vibratory equipment. Thus, we obtain:

$$
\left|\begin{array}{ccc}
c_{1}-m_{1} \cdot \omega^{2} & -c_{1} & 0 \\
-c_{1} & c_{1}+c_{2}-m_{2} \cdot \omega^{2} & -c_{2} \\
0 & -c_{2} & c_{2}-m_{3} \cdot \omega^{2}
\end{array}\right|=0 \text {, or }
$$

$-m_{1} \cdot m_{2} \cdot m_{3} \cdot \omega^{6}+\left(c_{1} \cdot m_{3} \cdot\left(m_{1}+m_{2}\right)+c_{2} \cdot m_{1} \cdot\left(m_{2}+m_{3}\right)\right) \cdot \omega^{4}-c_{1} \cdot c_{2} \cdot\left(m_{1}+m_{2}+m_{3}\right) \cdot \omega^{2}=0$.

The positive roots of the equation (8), i.e. the natural frequencies of the system's oscillations, obtained with a help of Wolfram Mathematica software are as follows:

$$
\begin{aligned}
& \omega_{n 1}=0 ; \\
& \omega_{n 2}=\sqrt{\frac{1}{2} \cdot \frac{\left[\begin{array}{c}
c_{1} \cdot\left(m_{1}+m_{2}\right) \cdot m_{3}+c_{2} \cdot m_{1} \cdot\left(m_{2}+m_{3}\right)- \\
-\sqrt{\left(c_{1} \cdot\left(m_{1}+m_{2}\right) \cdot m_{3}+c_{2} \cdot m_{1} \cdot\left(m_{2}+m_{3}\right)\right)^{2}-} \\
-4 \cdot c_{1} \cdot c_{2} \cdot m_{1} \cdot m_{2} \cdot m_{3} \cdot\left(m_{1}+m_{2}+m_{3}\right)
\end{array}\right]}{m_{1} \cdot m_{2} \cdot m_{3}}} ; \\
& \omega_{n 3}=\sqrt{\frac{1}{2} \cdot \frac{\left[\begin{array}{l}
c_{1} \cdot\left(m_{1}+m_{2}\right) \cdot m_{3}+c_{2} \cdot m_{1} \cdot\left(m_{2}+m_{3}\right)+ \\
+\sqrt{\left(c_{1} \cdot\left(m_{1}+m_{2}\right) \cdot m_{3}+c_{2} \cdot m_{1} \cdot\left(m_{2}+m_{3}\right)\right)^{2}-} \\
-4 \cdot c_{1} \cdot c_{2} \cdot m_{1} \cdot m_{2} \cdot m_{3} \cdot\left(m_{1}+m_{2}+m_{3}\right)
\end{array}\right]}{m_{1} \cdot m_{2} \cdot m_{3}}} .
\end{aligned}
$$

Solving the equations (6) with a help of the Kramer's rule, we obtain the dependencies for determining the amplitude values $X_{1}, X_{2}, X_{3}$ of the oscillating masses displacements:

$$
X_{1}=\frac{\operatorname{det}\left[\begin{array}{ccc}
0 & -c_{1} & 0 \\
F & c_{1}+c_{2}-m_{2} \cdot \omega^{2} & -c_{2} \\
-F & -c_{2} & c_{2}-m_{3} \cdot \omega^{2}
\end{array}\right]}{\operatorname{det}\left[\begin{array}{ccc}
c_{1}-m_{1} \cdot \omega^{2} & -c_{1} & 0 \\
-c_{1} & c_{1}+c_{2}-m_{2} \cdot \omega^{2} & -c_{2} \\
0 & -c_{2} & c_{2}-m_{3} \cdot \omega^{2}
\end{array}\right]}=
$$




$$
\begin{aligned}
& =\frac{-F \cdot c_{1} \cdot m_{3} \cdot \omega^{2}}{\left(c_{1}-m_{1} \cdot \omega^{2}\right) \cdot\left(c_{1}+c_{2}-m_{2} \cdot \omega^{2}\right) \cdot\left(c_{2}-m_{3} \cdot \omega^{2}\right)-c_{1}^{2} \cdot\left(c_{2}-m_{3} \cdot \omega^{2}\right)-c_{2}^{2} \cdot\left(c_{1}-m_{1} \cdot \omega^{2}\right)} \\
& X_{2}=\frac{\operatorname{det}\left[\begin{array}{ccc}
c_{1}-m_{1} \cdot \omega^{2} & 0 & 0 \\
-c_{1} & F & -c_{2} \\
0 & -F & c_{2}-m_{3} \cdot \omega^{2}
\end{array}\right]}{\operatorname{det}\left[\begin{array}{ccc}
c_{1}-m_{1} \cdot \omega^{2} & -c_{1} & 0 \\
-c_{1} & c_{1}+c_{2}-m_{2} \cdot \omega^{2} & -c_{2} \\
0 & -c_{2} & c_{2}-m_{3} \cdot \omega^{2}
\end{array}\right]}= \\
& =\frac{F \cdot\left(m_{1} \cdot \omega^{2}-c_{1}\right) \cdot m_{3} \cdot \omega^{2}}{\left(c_{1}-m_{1} \cdot \omega^{2}\right) \cdot\left(c_{1}+c_{2}-m_{2} \cdot \omega^{2}\right) \cdot\left(c_{2}-m_{3} \cdot \omega^{2}\right)-c_{1}^{2} \cdot\left(c_{2}-m_{3} \cdot \omega^{2}\right)-c_{2}^{2} \cdot\left(c_{1}-m_{1} \cdot \omega^{2}\right)}= \\
& =X_{1} \cdot\left(1-\frac{m_{1} \cdot \omega^{2}}{c_{1}}\right) \\
& X_{3}=\frac{\operatorname{det}\left[\begin{array}{ccc}
c_{1}-m_{1} \cdot \omega^{2} & -c_{1} & 0 \\
-c_{1} & c_{1}+c_{2}-m_{2} \cdot \omega^{2} & F \\
0 & -c_{2} & -F
\end{array}\right]}{\operatorname{det}\left[\begin{array}{ccc}
c_{1}-m_{1} \cdot \omega^{2} & -c_{1} & 0 \\
-c_{1} & c_{1}+c_{2}-m_{2} \cdot \omega^{2} & -c_{2} \\
0 & -c_{2} & c_{2}-m_{3} \cdot \omega^{2}
\end{array}\right]}= \\
& =\frac{F \cdot \omega^{2} \cdot\left(c_{1} \cdot\left(m_{1}+m_{2}\right)-m_{1} \cdot m_{2} \cdot \omega^{2}\right)}{\left(c_{1}-m_{1} \cdot \omega^{2}\right) \cdot\left(c_{1}+c_{2}-m_{2} \cdot \omega^{2}\right) \cdot\left(c_{2}-m_{3} \cdot \omega^{2}\right)-c_{1}^{2} \cdot\left(c_{2}-m_{3} \cdot \omega^{2}\right)-c_{2}^{2} \cdot\left(c_{1}-m_{1} \cdot \omega^{2}\right)}= \\
& =X_{2} \cdot \frac{c_{1} \cdot\left(m_{1}+m_{2}\right)-m_{1} \cdot m_{2} \cdot \omega^{2}}{\left(m_{1} \cdot \omega^{2}-c_{1}\right) \cdot m_{3}} \text {. }
\end{aligned}
$$

Thus, for the three-mass oscillatory system of vibratory finishing machine, we have the following group of parameters: $m_{1}, m_{2}, m_{3}, c_{1}, c_{2}, c_{i 3}, F, \omega, \varepsilon$. The values of the active $m_{1}$ and the intermediate $m_{2}$ masses (the masses of the upper and the lower lap, respectively) are to be given by the designer. The frequency $\omega$ and the initial phase $\varepsilon$ of the disturbing force are defined by the parameters of the electromagnetic drive. Therefore, the process of calculation of parameters of the oscillating systems of vibratory finishing machines can be reduced to determination of the values of the reactive mass $m_{3}$, the stiffness coefficients $c_{1}, c_{2}, c_{i 3}$, and the amplitude value of the disturbing force $F$. That is why, for the three-mass system of vibratory finishing machine, we have to determine five unknown parameters.

The stiffness $c_{i 3}$ can be chosen as relatively small one taking into account the condition that the mechanical oscillating system mounted (installed) on vibration isolators must have the self-frequency $\omega_{B}$ that is $n$ times smaller than the circular frequency of forced oscillations $\omega: \omega_{i 3}=\omega / n$. The larger is the value $n$, the weaker (safer) is the mechanical interaction between the oscillating system and the fixed frame of the machine. Its minimal value is $n=3 \ldots 4[11,13]$. Vibration isolators are to be calculated 


\section{Substantiation of Inertial, Stiffness and Excitation Parameters of Vibratory Lapping Machine ...}

according to the single-mass scheme consisting of one rigid body with the mass equal to the total mass of the whole structure and of the elastic element of certain stiffness representing the isolator. Thus, the stiffness coefficients of vibration isolators for three-mass systems can be determined as:

$$
c_{i 3}=\left(m_{1}+m_{2}+m_{3}\right) \cdot\left(\frac{\omega}{n}\right)^{2} .
$$

The equation (11) allow to derive the expression for calculating the stiffness of vibration isolators $c_{i 3}$. Based on this, we can state that there have left only four unknown parameters to be determined.

Let us analyse the characteristic equation (8) and equalize it to zero. Herewith, instead of the circular frequency $\omega$ let us substitute $\omega / z$ (where $\omega$ is the forced frequency of the system's oscillations; $z$ is the resonance setting-up (correction) coefficient):

$$
\begin{aligned}
-m_{1} \cdot m_{2} \cdot m_{3} \cdot\left(\frac{\omega}{z}\right)^{4} & +\left(c_{1} \cdot m_{3} \cdot\left(m_{1}+m_{2}\right)+c_{2} \cdot m_{1} \cdot\left(m_{2}+m_{3}\right)\right) \cdot\left(\frac{\omega}{z}\right)^{2}- \\
& -c_{1} \cdot c_{2} \cdot\left(m_{1}+m_{2}+m_{3}\right)=0 .
\end{aligned}
$$

The resonance correction coefficient $z$ is usually in the range of [0.93...0.98] and provides the setting-up of the machine operation in near-resonance mode concerning the third natural frequency $\omega_{n 3}$

Using the equation (12), let us determine the expression for calculating the stiffness $c_{2}$ of the elastic element connecting the masses $m_{2}$ and $m_{3}$ :

$$
c_{2}=m_{3} \cdot\left(\frac{\omega}{z}\right)^{2} \cdot \frac{c_{1} \cdot\left(m_{1}+m_{2}\right)-m_{1} \cdot m_{2} \cdot\left(\frac{\omega}{z}\right)^{2}}{c_{1} \cdot\left(m_{1}+m_{2}+m_{3}\right)-m_{1} \cdot\left(m_{2}+m_{3}\right) \cdot\left(\frac{\omega}{z}\right)^{2}} .
$$

The condition of providing the in-phase motion of the reactive and the intermediate masses allows minimization of the push-pull force of electromagnet and minimization of the consumed power since the air gap between the armature and the core of electromagnet remains unchanged; it does not depend on the relative amplitudes of oscillations of the corresponding masses. In this case the masses move at the same phases and with equal amplitudes like one rigid body. In order to excite the vibrations of the system, it is necessary to use push-pull (two-cycle, two-stroke) electromagnets whose cores are attached to one mass (for example, to the lower lap), and armatures - to the other one. For the purpose of implementation of the considered condition let us equalize the second and the third analytical expressions of the system (10) and determine the value of the reactive mass $m_{3}$ :

$$
\begin{gathered}
X_{2}=X_{3} \Rightarrow \frac{F \cdot\left(m_{1} \cdot \omega^{2}-c_{1}\right) \cdot m_{3} \cdot \omega^{2}}{\left[\begin{array}{c}
\left(c_{1}-m_{1} \cdot \omega^{2}\right) \cdot\left(c_{1}+c_{2}-m_{2} \cdot \omega^{2}\right) \cdot\left(c_{2}-m_{3} \cdot \omega^{2}\right)- \\
-c_{1}^{2} \cdot\left(c_{2}-m_{3} \cdot \omega^{2}\right)-c_{2}^{2} \cdot\left(c_{1}-m_{1} \cdot \omega^{2}\right)
\end{array}\right]}= \\
=\frac{F \cdot \omega^{2} \cdot\left(c_{1} \cdot\left(m_{1}+m_{2}\right)-m_{1} \cdot m_{2} \cdot \omega^{2}\right)}{\left[\begin{array}{c}
\left(c_{1}-m_{1} \cdot \omega^{2}\right) \cdot\left(c_{1}+c_{2}-m_{2} \cdot\left(\frac{\omega}{z}\right)^{2}\right) \cdot\left(c_{2}-m_{3} \cdot \omega^{2}\right)- \\
-c_{1}^{2} \cdot\left(c_{2}-m_{3} \cdot \omega^{2}\right)-c_{2}^{2} \cdot\left(c_{1}-m_{1} \cdot \omega^{2}\right)
\end{array}\right]} \Rightarrow
\end{gathered}
$$




$$
\begin{gathered}
\Rightarrow X_{2}=X_{2} \cdot \frac{c_{1} \cdot\left(m_{1}+m_{2}\right)-m_{1} \cdot m_{2} \cdot \omega^{2}}{\left(m_{1} \cdot \omega^{2}-c_{1}\right) \cdot m_{3}} \Rightarrow \frac{c_{1} \cdot\left(m_{1}+m_{2}\right)-m_{1} \cdot m_{2} \cdot \omega^{2}}{\left(m_{1} \cdot \omega^{2}-c_{1}\right) \cdot m_{3}}=1 \Rightarrow \\
\Rightarrow m_{3}=\frac{c_{1} \cdot\left(m_{1}+m_{2}\right)-m_{1} \cdot m_{2} \cdot \omega^{2}}{m_{1} \cdot \omega^{2}-c_{1}} .
\end{gathered}
$$

The condition of providing the anti-phase motion of the upper and the lower laps of vibratory finishing machine allows maximization of the relative speed of the laps' motion and of the intensity of their dressing (or in other words, minimization of duration of the dressing process). In this case, the masses (the upper and the lower laps) move at the opposite phases and with equal amplitudes. For the purpose of implementation of the considered condition let us add the first and the second analytical expressions of the system (10), equalize the obtained sum to zero and determine the value of the stiffness $c_{1}$ :

$$
\left.\begin{array}{c}
X_{1}+X_{2}=0 \Rightarrow \frac{-F \cdot c_{1} \cdot m_{3} \cdot \omega^{2}}{\left[\left(c_{1}-m_{1} \cdot \omega^{2}\right) \cdot\left(c_{1}+c_{2}-m_{2} \cdot \omega^{2}\right) \cdot\left(c_{2}-m_{3} \cdot \omega^{2}\right)-\right.} \\
-c_{1}^{2} \cdot\left(c_{2}-m_{3} \cdot \omega^{2}\right)-c_{2}^{2} \cdot\left(c_{1}-m_{1} \cdot \omega^{2}\right)
\end{array}\right]+
$$

Taking into account the necessity to provide the prescribed (technologically determined) amplitudes of the laps' oscillations while carrying out their dressing, let us denote the amplitude value of the upper lap displacement as $d_{1}$. Thus, we can equalize this value to $X_{1}$ (see formula (10)) and determine the necessary excitation force of electromagnetic drive:

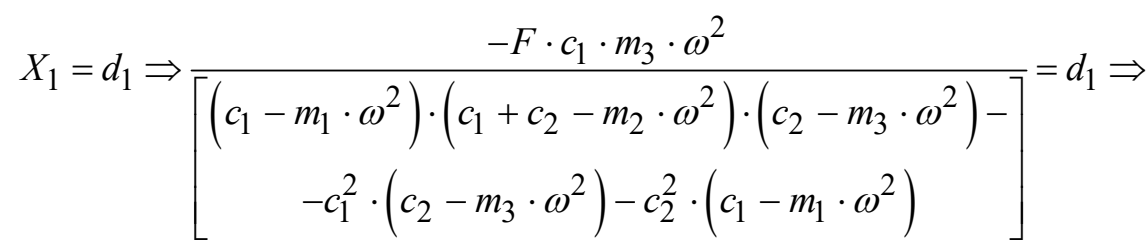

$$
\begin{aligned}
& \Rightarrow F=\frac{-d_{1} \cdot\left[\begin{array}{c}
\left(c_{1}-m_{1} \cdot \omega^{2}\right) \cdot\left(c_{1}+c_{2}-m_{2} \cdot \omega^{2}\right) \cdot\left(c_{2}-m_{3} \cdot \omega^{2}\right)- \\
-c_{1}^{2} \cdot\left(c_{2}-m_{3} \cdot \omega^{2}\right)-c_{2}^{2} \cdot\left(c_{1}-m_{1} \cdot \omega^{2}\right)
\end{array}\right]}{c_{1} \cdot m_{3} \cdot \omega^{2}} .
\end{aligned}
$$

Solving the system of four equations (12) - (16) in Wolfram Mathematica software, we can define four unknown inertial, stiffness and excitation parameters $m_{3}, c_{1}, c_{2}, F$. The fragment of Wolfram Mathematica window with the solution of the system of equations (12) - (16) is presented in Fig. 2. Thus, for calculating (analysing) the parameters of the mechanical oscillating system of vibratory finishing machine we can use the following formulas:

$$
\begin{aligned}
& m_{3}=m_{1}-m_{2} \\
& c_{1}=\frac{m_{1} \cdot \omega^{2}}{2}
\end{aligned}
$$


Substantiation of Inertial, Stiffness and Excitation Parameters of Vibratory Lapping Machine ...

$$
\begin{gathered}
c_{2}=\frac{\left(m_{1}-m_{2}\right) \cdot \omega^{2} \cdot\left(m_{1} \cdot z^{2}+m_{2} \cdot\left(z^{2}-2\right)\right)}{2 \cdot m_{1} \cdot z^{2} \cdot\left(z^{2}-1\right)} ; \\
F=d_{1} \cdot\left(m_{2}-m_{1}\right) \cdot \omega^{2} .
\end{gathered}
$$

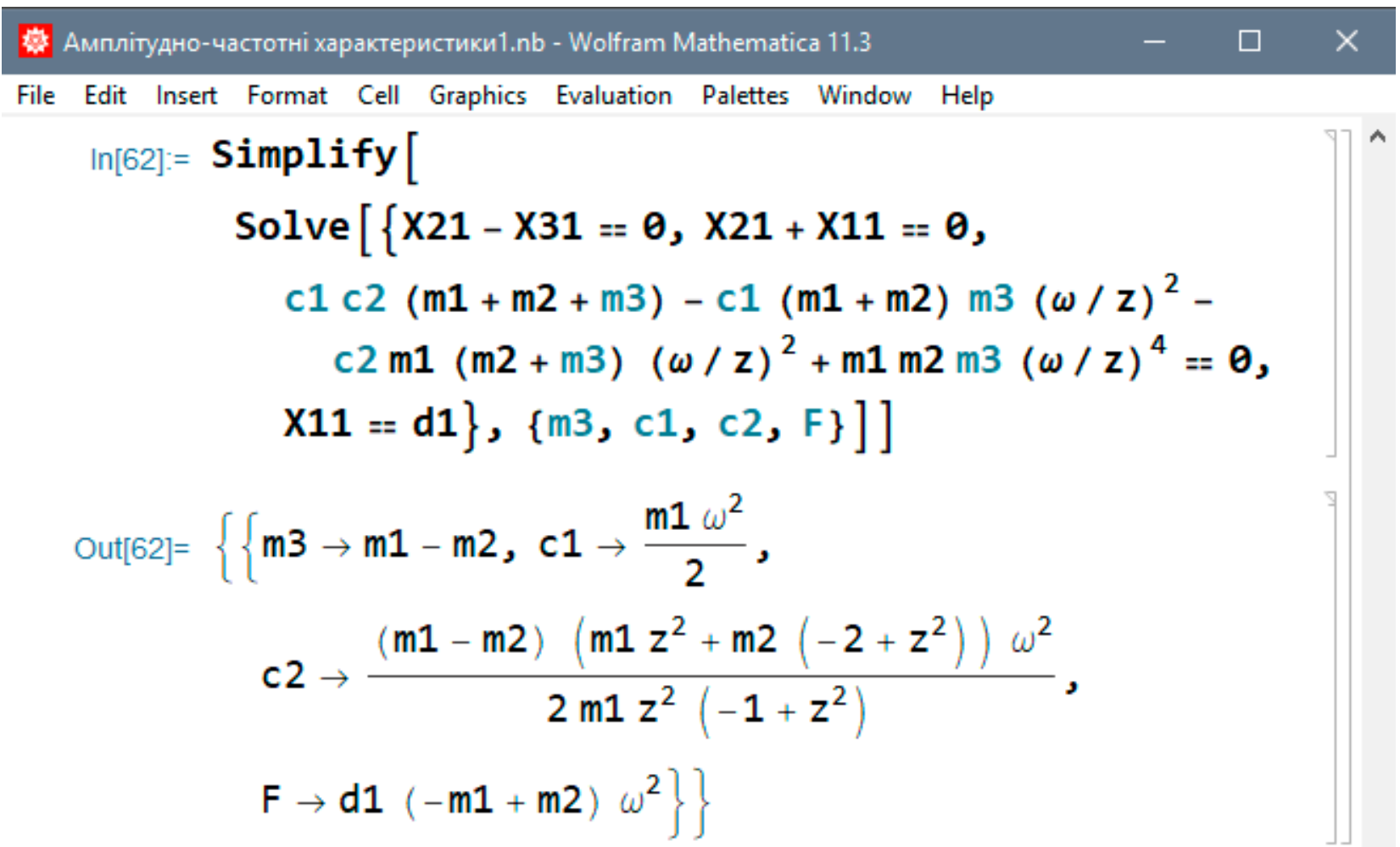

Fig. 2. Fragment of Wolfram Mathematica window with the solution of the system of equations (12) - (16)

In order to find the steady-state solution of the differential equations (1) and to simulate the system's motion, let us prescribe the parameters of the oscillating system of vibratory lapping machine: $m_{1}=3.32 \mathrm{~kg}, m_{2}=3.04 \mathrm{~kg}, \omega=314 \frac{\mathrm{rad}}{\mathrm{s}}$, and some operation parameters $z=0.96, d_{1}=-0.003 \mathrm{~m}$. Substituting theses values into the formulas (17), we calculate the corresponding unknown parameters: $c_{1} \approx 163669 \frac{\mathrm{N}}{\mathrm{m}}, c_{2} \approx 12580 \frac{\mathrm{N}}{\mathrm{m}}, m_{3} \approx 0.28 \mathrm{~kg}, F \approx 83 \mathrm{H}$. Since $c_{2} \ll c_{1}$, we have proved the assumption about "zero stiffness" phenomenon and in-phase motion of reactive and intermediate masses $[11,13]$. The obtained values ensure one of the most efficient modes of "lap over lap" dressing. Herewith, the oscillating system uses the effect of "zero-stiffness" when the reactive mass and the lower lap move at the same phase with equal amplitudes. Due to occurring the in-phase motion, the air gap in electromagnetic vibration exciter can be minimal because in such a case there is no relative displacements between two adjacent masses. This allows significant decreasing of the value of current flowing in the winding of the vibration exciter, as well as the power consumed $[11,13]$.

On the basis of the given input data and the obtained results of theoretical investigations (17), using the derived expressions (3) and (10), let us plot the amplitude-frequency characteristics (Fig. 3) and the time dependencies of displacements of the oscillating masses in their steady-state motion (Fig. 4, a).

In order to confirm the theoretical approaches presented in the paper, there was carried out a numerical solving of the system of differential equations (1) using the corresponding built-in function "DSolve" of Wolfram Mathematica software (Fig. 4, b). 


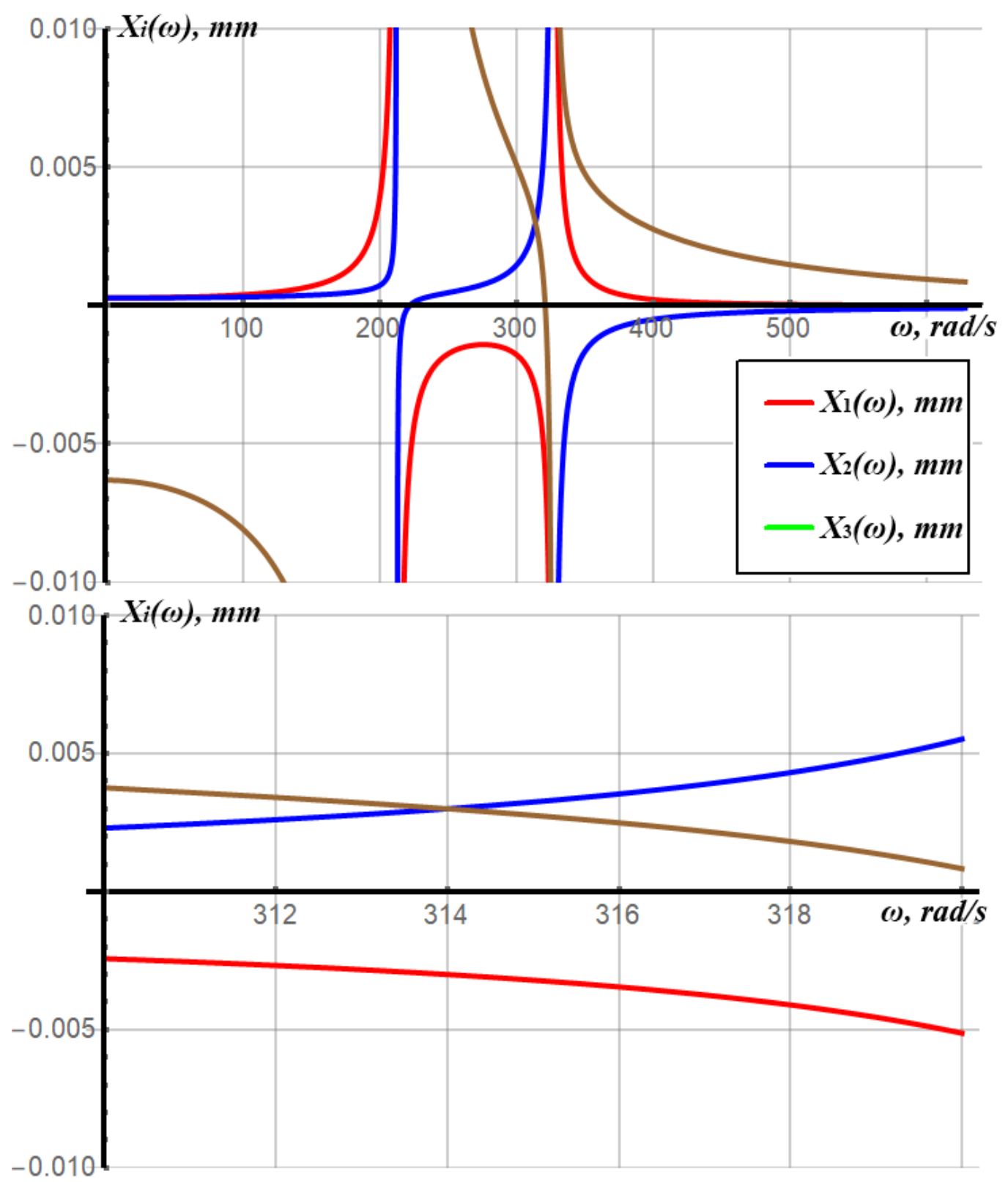

Fig. 3. Amplitude-frequency characteristics of the three-mass oscillating system of vibratory finishing machine

Analysing the obtained amplitude-frequency characteristics (Fig 3) and time dependencies of oscillations of masses of vibratory finishing machine (Fig 4) within the range of frequencies close to resonance (in particular, at the frequency of $314 \mathrm{rad} / \mathrm{s}$ ), we have to mention the equality of the amplitudes of oscillations of the intermediate mass (lower lap) and the reactive mass (electromagnet's armature) (about $3 \mathrm{~mm}$ ). This states about their in-phase motion and the realization of "zero-stiffness" effect. The oscillations of the active mass (upper lap) are at the opposite phase to the oscillations of the other two masses, and the amplitude of the upper lap oscillations is almost equal to the amplitudes of the other masses (Figs. 3 -4). This is confirmed by the results of modelling (simulation) of the oscillating masses motion using the analytical dependencies (3) and by the results of numerical solving of the system of differential equations of the oscillating masses motion in Wolfram Mathematica software (Fig. 4). Approximately at $0.05 \mathrm{~s}$ after the system is started (switched on), one can see the synchronous steady-state oscillations of the intermediate mass at the opposite phase to the motion of the active mass (Fig. 4, b). Herewith, the amplitudes of oscillations of the intermediate and the reactive masses become equal approximately at $0.15 \mathrm{~s}$ after the system is started (Fig. 4, b). It is necessary to mention that some 
Substantiation of Inertial, Stiffness and Excitation Parameters of Vibratory Lapping Machine ... differences between the amplitude values of displacements in Figs. 4 a and $b$ can be explained by taking into account viscous friction (damping) while carrying out the simulation modelling.
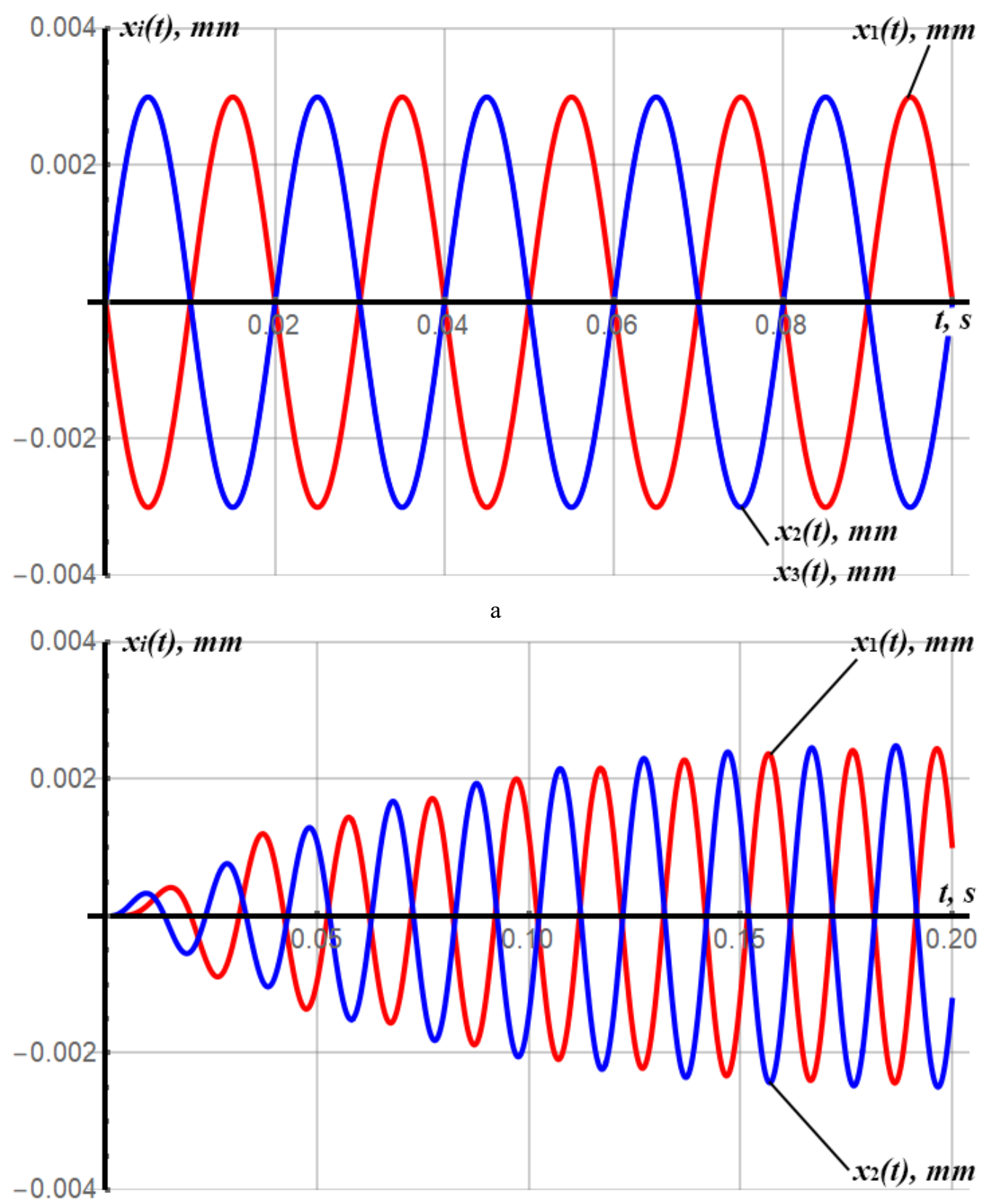

$\mathrm{b}$

Fig. 4. Time dependencies of the oscillating masses displacements: a - constructed on the basis of the formulas (3); $\mathrm{b}$ - constructed using the results of numerical solving of the system of differential equations (1) in Wolfram Mathematica software

Therefore, the derived expressions (17) for determining the inertial, stiffness and excitation parameters of the three-mass mechanical oscillating system of vibratory finishing machine ensure its highest efficiency and the largest intensity of the laps dressing process because of the antiphase motion of the laps, and of the absence of the relative motion between the reactive and intermediate masses.

\section{Conclusions}

In the paper, there were overviewed the prospects of use and development of vibratory lapping machines. Based on the carried out analysis of modern information sources on the subject of the paper, the 


\section{Ihor Kuzio, Viktor Zakharov, Vitaliy Korendiy}

main investigations were focused on derivation of analytical dependencies for calculating the inertial, stiffness and excitation parameters of mechanical oscillating system of vibratory finishing machine in order to ensure its energy-efficient resonance operation mode.

The simplified (two-dimensional) design diagram of mechanical oscillatory system of vibratory finishing machine was developed (see Fig. 1). Using the Lagrange's equations, the mathematical model (1) of the system's dynamics was derived. Using the general methods of solving the obtained system of differential equations, the expressions for calculating the natural frequencies of the oscillating system (9) and the amplitudes of the oscillating masses' displacements (10) were deduced. Based on the substantiated assumptions, the analytical dependencies (13)-(17) for calculating inertial, stiffness and excitation parameters of the system were derived.

In order to simulate the laps' motion, using the prescribed parameters of the oscillating system, there were determined the stiffness coefficients of elastic elements $c_{1} \approx 163669 \frac{\mathrm{N}}{\mathrm{m}}, c_{2} \approx 12580 \frac{\mathrm{N}}{\mathrm{m}}$, the value of the reactive mass $m_{3} \approx 0.28 \mathrm{~kg}$, and the amplitude value of the excitation force $F \approx 83 \mathrm{H}$. It was substantiated that these parameters ensure one of the most efficient modes of "lap over lap" dressing using "zero stiffness" phenomenon and in-phase motion of reactive and intermediate masses.

The results of the conducted theoretical investigations (Fig. 3) were compared with the results of simulation modelling of the system's motion carried out with a help of Wolfram Mathematica software (Fig. 4). The antiphase motion of the upper and the lower laps with equal amplitudes was proved, whence the reactive mass vibrated at the same phase and with the same amplitude as the intermediate mass did.

The results of the carried out investigations can be used while developing new structures and improving existent designs of vibratory lapping machines. The scopes of further investigations consist in analysing three-dimensional diagrams of vibratory finishing machines, investigating the dynamical properties of their mechanical oscillating systems and substantiating the possibilities of ensuring circular (two-dimensional) oscillations of the laps while carrying out the processes of their dressing and lapping flat surfaces of cylindrical and prismatic machine parts.

\section{References}

[1] R. Mediratta, K. Ahluwalia, and S. H. Yeo, "State-of-the-art on vibratory finishing in the aviation industry: an industrial and academic perspective," Int. J. Adv. Manuf. Technol., vol. 85, no. 1-4, pp. 415-429, Jul. 2016.

[2] D. Huo, and F. Wardle, "A holistic integrated dynamic design and modelling approach applied to the development of ultraprecision micro-milling machines," Int. J. Mach. Tools Manuf., vol. 50, no. 4, pp. 335-343, Apr. 2010.

[3] V. Tsiakoumis, and A. Batako, "Vibration-Assisted Grinding of Mild and Hardened Steel: A Novel Design Vibrating Jig and Process Performance," in Volume 4: Advanced Manufacturing Processes; Biomedical Engineering; Multiscale Mechanics of Biological Tissues; Sciences, Engineering and Education; Multiphysics; Emerging Technologies for Inspection, 2012, pp. 141-146.

[4] J. Yuan, W. Yao, P. Zhao, B. Lyu, Z. Chen, and M. Zhong, "Kinematics and trajectory of both-sides cylindrical lapping process in planetary motion type," Int. J. Mach. Tools Manuf., vol. 92, pp. 60-71, May 2015.

[5] D. Wen, H. Qi, L. Ma, C. Lu, and G. Li, "Kinematics and trajectory analysis of the fixed abrasive lapping process in machining of interdigitated micro-channels on bipolar plates," Precis. Eng., vol. 44, pp. 192-202, Apr. 2016.

[6] F. Hashimoto, and D. B. DeBra, "Modelling and Optimization of Vibratory Finishing Process," CIRP Ann., vol. 45, no. 1, pp. 303-306, Jan. 1996.

[7] F. Hashimoto, and S. P. Johnson, "Modeling of vibratory finishing machines," CIRP Ann., vol. 64, no. 1, pp. 345-348, Jan. 2015.

[8] Y. B. Tian, Z. W. Zhong, and S .J. Tan, "Kinematic analysis and experimental investigation on vibratory finishing," Int. J. Adv. Manuf. Technol., vol. 86, no. 9-12, pp. 3113-3121, October 2016.

[9] P. P. Kumar, and S. Sathyan, "Simulation of 1D Abrasive Vibratory Finishing Process," Adv. Mater. Res., vol. 565, pp. 290-295, Sep. 2012.

[10] O. V. Havrylchenko, V. M. Korendiy, and V. M. Zakharov, "Formuvannia matematychnykh modelei tryi chotyrymasovykh kolyvnykh system vibrovykinchuvalnykh verstativ" ["Formation mathematical model three and 
Substantiation of Inertial, Stiffness and Excitation Parameters of Vibratory Lapping Machine ...

four mass oscillatory systems of vibrating finishing machines"], Visnyk Natsionalnoho universytetu "Lvivska politekhnika" [Bulletin of Lviv Polytechnic National University], no. 866, p. 3-12, 2017. [in Ukrainian].

[11] V. Korendiy, and V. Zakharov, "Substantiation of Parameters and Analysis of Operational Characteristics of Oscillating Systems of Vibratory Finishing Machines", Ukrainian Journal of Mechanical Engineering and Materials Science, vol. 3, no. 2, pp. 67-78, 2017.

[12] I. V. Kuzio, V. M. Zakharov, and V. M. Korendiy, "Modelling the process of dressing the laps of vibratory finishing machine", Avtomatizaciâ virobničih procesìv u mašinobuduvannì ta priladobuduvannì [Industrial Process Automation in Engineering and Instrumentation], no. 52, p. 81-93, 2018.

[13] O. Lanets, Osnovy rozrakhunku ta konstruiuvannia vibratsiinykh mashyn [Fundamentals of Analysis and Design of Vibratory Machines], Lviv, Ukraine: Lviv Polytechnic Publishing House, 2018. [in Ukrainian].

[14] V. M. Gursky, Bahatokryterialnyi analiz i syntez neliniinykh rezonansnykh vibratsiinykh mashyn [MultiCriteria Analysis and Synthesis of the Nonlinear Resonant Vibratory Machines], Lviv, Ukraine: Lviv Polytechnic Publishing House, 2017. [in Ukrainian]. 